\title{
Acuidade visual em implantes bilaterais de lentes intra-oculares monofocais e multifocais
}

\author{
Visual acuity ofbilateral implants of monofocal and multifocal intraocular lenses
}

\author{
Leonardo Akaishi ${ }^{1}$ \\ André Gustavo Rolim de Araújo ${ }^{2}$ \\ Regina C. N. dos Santos ${ }^{3}$ \\ Procópio Miguel dos Santos ${ }^{4}$
}

Tese apresentada ao Curso de Pós-Graduação em Ciências da Saúde, Universidade de Brasília (UNB), área de concentração Oftalmologia, para obtenção do título de mestre.

Trabalho realizado no Setor de Catarata e Cirurgia do Segmento Anterior do Hospital Oftalmológico de Brasília (HOB).

Oftalmologista do Setor de Catarata e Cirurgia do Segmento Anterior e Diretor do Hospital Oftalmológico de Brasília (HOB), mestrando pela Faculdade de Ciências da Saúde da Universidade de Brasília (UNB).

2 Oftalmologista do Setor de Catarata e Cirurgia do Segmento Anterior do Hospital Oftalmológico de Brasília (HOB).

${ }^{3}$ Professora orientadora do Decanato de Pós-Graduação da UNB e Preceptora de Residência Médica do Hospital de Base do Distrito Federal (HBDF).

${ }^{4}$ Professor orientador do Decanato de Pós-Graduação da Universidade de Brasília e Coordenador da Residência Médica do Hospital de Base do Distrito Federal (HBDF).

Endereço para correspondência: Leonardo Akaishi Hospital Oftalmológico de Brasília, SGAS, 607, L2 sul, Plano Piloto - Brasília (DF)

Recebido para publicação em 29.01.2002 Aceito para publicação em 12.06.2002

\begin{tabular}{|l|}
\hline RESUMO \\
\hline Objetivo: Comparar a acuidade visual de longe e perto de pacientes \\
submetidos à facoemulsificação binocularnão simultânea com implante de \\
lentes multifocais ou monofocais. Métodos: Foram selecionados 20 pa- \\
cientes com lentes multifocais bilaterais e outros 20 pacientes com lentes \\
monofocais também bilaterais, com acuidade visual sem correção melhor \\
ou igual a 0,63 (20/30), medidos separadamente, nos três primeiros meses \\
de pós-operatório. Foi medida a acuidade visual para longe e perto com e \\
sem correção e testes de sensibilidade ao contraste e ofuscamento. \\
Resultados: A acuidade visual sem correção para longe no grupo das \\
monofocais teve média de 0,82 (DP \pm , 16 ) no grupo das multifocais, 0,94 \\
(DP $\pm 0,12$ ), valor de p 0,001. Os dois grupos de lentes tiveram visão com \\
correção para longe igual a 1. No grupo das multifocais, 75\% tiveram J1 e \\
100\% tiveram J3 ou melhor sem correção. No grupo das lentes monofocais, \\
$10 \%$ tiveram J1 e $70 \%$ tiveram J3 ou melhor sem correção. Não houve \\
diferença significante na avaliação com o teste de sensibilidade ao contras- \\
te entre os grupos pesquisados. No teste de ofuscamento, os dois grupos \\
tiveram redução da visão, que foi mais acentuada no grupo dos pacientes \\
com lentes multifocais. Conclusão: A acuidade visual para longe com \\
correção nos pacientes com implante multifocal foi semelhante a dos \\
pacientes com implantes monofocais, embora acuidade visual para perto \\
no grupo em que foi implantado lente multifocal foi bastante superior ao \\
grupo da lente monofocal. A sensibilidade ao contraste manteve-se \\
semelhante nos dois grupos, já o ofuscamento ("glare test") no grupo \\
multifocal foi maior que no grupo monofocal. \\
\hline
\end{tabular}

Descritores: Acuidade visual; Implante de lente intra-ocular; Lentes intra-oculares; Facoemulsificação; Adulto

\section{INTRODUÇ̃̃̃O}

Estima-se que a catarata represente mais de 50\% dos 40 milhões de cegos no mundo, sendo considerada a causa mais comum de cegueira. Levantamentos recentes realizados no Brasil calculam uma população de 140 mil cegos e 700 mil deficientes visuais por catarata não operada. Para um número tão vultoso tem-se uma estimativa de 90 mil cirurgias de catarata realizadas no Brasil por ano ${ }^{(1)}$. Desde a aprovação da lente intra-ocular multifocal progressiva AMO Array pelo FDA, que proporciona uma visão para longe, intermediária e perto, tem sido estudada a importância dos melhoramentos na qualidade de vida, ou seja, proporcionar ao paciente enxergar sem o uso dos óculos ${ }^{(2)}$. Embora de uso crescente, o implante das lentes multifocais também tem gerado relatos de problemas visuais indesejáveis como halos e "glare" noturno. 
O objetivo deste trabalho foi avaliar a acuidade visual para longe e perto, a sensibilidade ao contraste e ofuscamento dos pacientes submetidos à facoemulsificação com implantes bilaterais não simultâneos de lentes intra-oculares monofocais, comparados aos pacientes com implantes intra-oculares multifocais (SA40NI Allergan).

\section{MÉTODOS}

Foram incluídos 40 pacientes submetidos à facoemulsificação com implantes de lentes intra-oculares multifocais e monofocais bilateral no Setor de Catarata do Hospital Oftalmológico de Brasília (HOB). Estes pacientes necessariamente deveriam ter em ambos olhos lentes monofocais ou lentes multifocais. A acuidade visual mínima necessária, sem correção, deveria ser de 20/30 na escala de Snellen ou 0,63 na escala decimal em ambos olhos, medidos separadamente nos 3 primeiros meses de pós-operatório. As lentes deveriam estar centralizadas e a cápsula posterior do cristalino intacta e transparente.

Os pacientes foram divididos em dois grupos: 20 pacientes ( 40 olhos) sendo 16 mulheres e 4 homens com implantes bilaterais de lentes monofocais (SI40 Allergan); 20 pacientes (40 olhos) sendo 15 mulheres e 5 homens com implantes bilaterais de lentes multifocais (SA40NI Allergan).

Foram excluídos os pacientes que apresentavam: acuidade visual no pós-operatório pior do que 0,63 na escala decimal ou 20/30 na escala de Snellen, em qualquer dos olhos medidos, sem correção nos 3 meses de acompanhamento; aparecimento de qualquer doença ocular ou sistêmica que poderia comprometer a acuidade visual nos 3 primeiros meses de pós-operatório; complicações intra ou pós-operatórias; astigmatismo pósoperatório maior que 1,00 topograficamente e/ou refracional; descentrações de lentes intra-oculares.

Cada paciente, antes da cirurgia, foi submetido à avaliação da história clínica e exame oftalmológico completo, pelo mesmo pesquisador. O exame constou de medida da acuidade visual, biomicroscopia, topografia corneana, tonometria de aplanação, oftalmoscopia indireta e, por último, ecobiometria. Para os cálculos das lentes, foi utilizada a fórmula SRK/T e, para os olhos menores de $21 \mathrm{~mm}$ (diâmetro ântero-posterior), foi utilizada a fórmula de Holladay 1. O poder das lentes a serem implantadas era selecionado objetivando a emetropia, com discreta tendência à miopização no grupo das monofocais e discreta tendência à hipermetropização no grupo das multifocais.

A acuidade visual para longe foi testada a 6 metros com correção e sem correção monocularmente, convertendo a escala de Snellen para escala decimal (0,05 a 1,0 igual 10/200 a 20/20) e para cálculo estatístico. A acuidade para perto foi medida binocularmente sem correção e com a melhor correção a $40 \mathrm{~cm}$ de distância com uma lâmpada de $40 \mathrm{w}$, usando a tabela de Jaeger $(\mathrm{J} 1=1,0 ; \mathrm{J} 2=0,8 ;$ etc. $)$ e convertendo para a escala decimal. O teste de sensibilidade ao contraste e o teste de ofus- camento foram realizados entre 90 e 100 dias pós-operatórios feita de acordo com as especificações do fabricante, usando a acuidade de luminosidade do aparelho CSV-1000 (Vector Vision, Ohio, EUA). Os testes foram feitos com a melhor correção para longe numa tabela projetada a 3 metros, conforme especificação do manual do fabricante (Figura 1). Os exames oftalmológicos foram realizados no $1^{\circ}, 15^{\circ}, 30^{\circ}, 60^{\circ}$ e $90^{\circ}$ dia após a cirurgia. As cirurgias foram realizadas pelo mesmo cirurgião no período entre agosto de 1999 a outubro de 2000.

No pré-operatório os pacientes utilizaram colírio composto de ciprofloxacina associado à dexametasona (Allergan), de $8 \mathrm{em}$ 8 horas, dois dias antes da cirurgia. Foi realizada uma dilatação pupilar, aproximadamente 30 minutos antes da cirurgia, com instilação de solução de tropicamida (Alcon, USA) 1\% e cloridrato de fenilefrina (Alcon, USA) 10\%, numa proporção de 1:1 (uma gota de 5 em 5 minutos, por 3 vezes). A anestesia, em todos os casos, foi tópica, com instilação de cloridrato de lidocaína geléia a 2\% (Astra Zeneca, Argentina), sem vasoconstritor no fundo de saco conjuntival, 30 minutos antes da

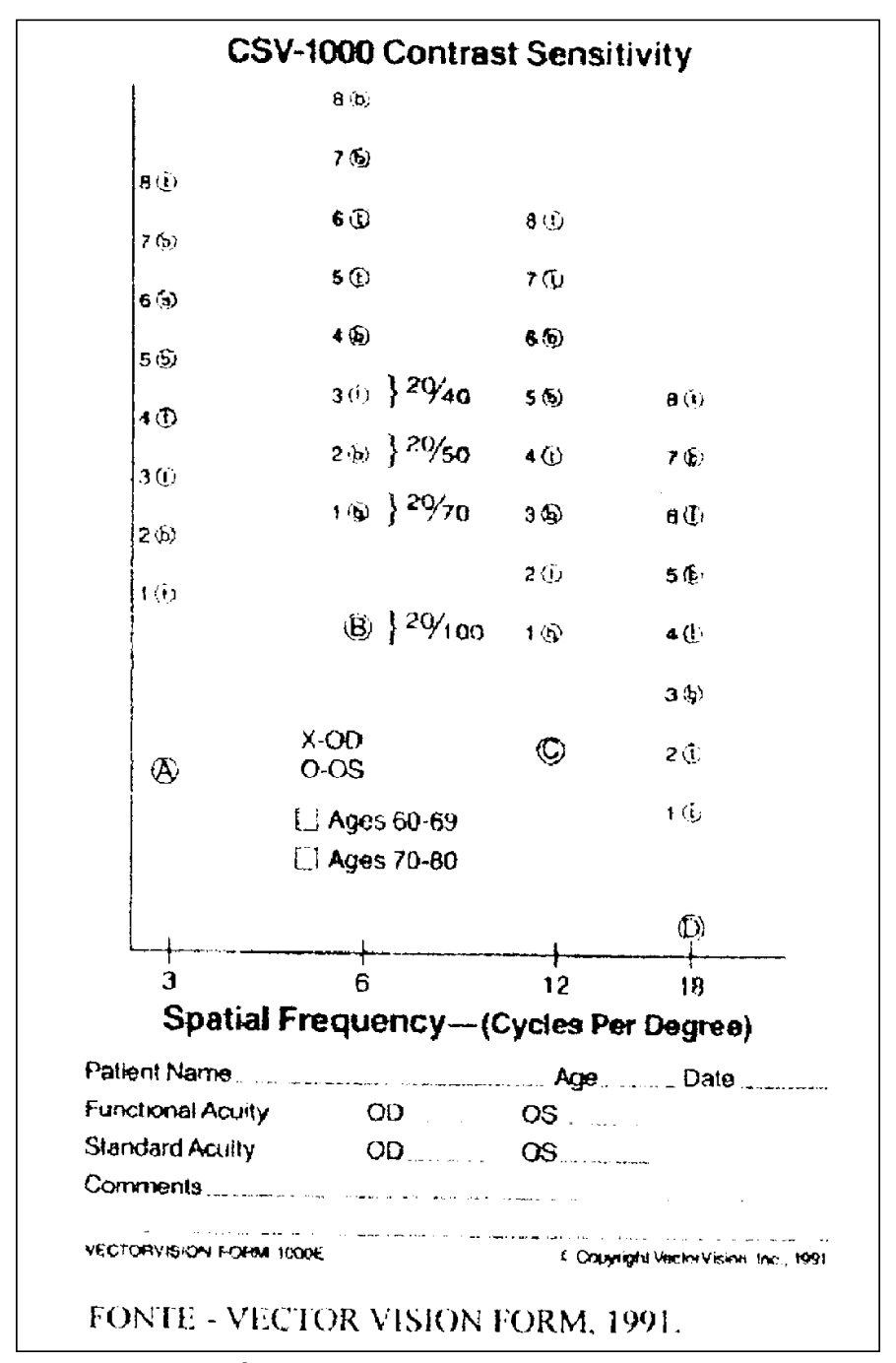

Figura 1 - Formulário do teste de sensibilidade ao contraste 
cirurgia, por 2 vezes em um intervalo de 10 minutos. Foi utilizada também 1 gota de cloridrato de proximetacaína (Alcon, USA), antes do cloridrato de lidocaína. Por via endovenosa, utilizouse $1,0 \mathrm{mg}$ de midazolam (Roche, Brasil) $5 \mathrm{mg} / \mathrm{ml}$.

Após a instilação do anestésico, foi realizada limpeza ocular com soro fisiológico a $0,9 \%$ (Halex Istar, Brasil) para remoção do resto do anestésico e, em seguida, anti-sepsia com solução de povidine tópico (Cinord Sul, Brasil) e a instilação de 1 gota de povidine a 3\% (Ophthalmos, Brasil). Foi realizada paracentese de $0,8 \mathrm{~mm}$ de tamanho. Em todos os casos, a incisão foi temporal na córnea transparente com sulco prévio de $300 \mu$ de profundidade e $2,8 \mathrm{~mm}$ de extensão com lâmina de diamante. A troca de humor aquoso foi feita por substância de hialuronato de sódio (Alcon, USA) e, com cistítomo, realizouse o início da capsulorrexe que foi terminada com pinça de Utrata no sentido anti-horário. A capsulorrexe era de tamanho de $5,5 \mathrm{~mm}$ a $6 \mathrm{~mm}$ de diâmetro. Utilizando uma cânula de calibre 23 e solução balanceada salina, foram realizadas hidrodissecção e hidrodelineação, quando possível. Para a emulsificação do núcleo, foram utilizados ponteira tipo Kelman de 30 graus, ABS e aparelho Legacy da Alcon (USA). Após a realização do sulco vertical, o núcleo foi dividido em 2 partes e realizada a emulsificação das metades do núcleo pelas técnicas de "Stop and Chop" e Dividir e Conquistar. Todo o córtex do cristalino foi removido por uma caneta de aspiração-irrigação automatizada. Após o término deste processo, iniciou-se a infusão de metilcelulose a 2\% (Alcon, USA) para preencher o saco capsular, facilitando assim, a introdução da LIO. Em todos as cirurgias, as LIOs foram implantadas no saco capsular por meio de injetores (Allergan, USA), com as alças no sentido das 3-9h. Concluído o implante da LIO, era aspirado todo o viscoelástico remanescente com irrigação-aspiração automatizada, com devida reposição de solução balanceada e injeção no estroma da incisão da córnea a fím de causar um edema estromal e tornar a incisão auto-selante. Ao final da cirurgia certificou-se de que a coaptação das bordas da incisão era satisfatória e auto-selante. Foi instilada 1 gota de colírio composto de cloridrato de ciprofloxacina associada a dexametasona (Allergan, USA). Em nenhuma das cirurgias foi necessário o uso de curativos.

Após a cirurgia, os pacientes eram orientados quanto à utilização dos seguintes medicamentos: cloridrato de ciprofloxacina associado à dexametasona (Allergan, USA) 4 vezes ao dia (6/6h), nos primeiros 15 dias e dextrano 70-hipromelose (Alcon, USA) 3 vezes ao dia, por 30 dias. Após 15 dias, o cloridrato de ciprofloxacina e a dexametasona (Allergan, USA) eram substituídos por acetato de fluormetolona $0,1 \%$ (Alcon, USA), 3 vezes ao dia, por 15 dias. Decorrido este período, suspendia-se o acetato de fluormetolona 0,1\% (Alcon, USA) de forma progressiva: 2 vezes ao dia por 7 dias e 1 vez ao dia por mais 7 dias. Assim ao final do $45^{\circ}$ dia do pós-operatório, foram suspensos todos os colírios.

Para a análise dos resultados, foi aplicado o teste $t$ student, o qual permitiu determinar se as médias dos dois grupos de observações foram estatisticamente diferentes ${ }^{(3)}$.

\section{RESULTADOS}

A amostra foi formada em sua maioria por mulheres (4 homens e 16 mulheres no grupo das multifocais e 5 homens e 15 mulheres no grupo das monofocais). A idade dos indivíduos submetidos a lentes monofocais variou entre 54 e 80 anos, com uma média de 68 anos (mediana 70 anos). No grupo das lentes multifocais, a idade variou entre 53 e 85 anos, com uma média de 63 anos (mediana 61 anos).

Outra característica avaliada foi a refração (equivalente esférico). Os dados avaliados nas Tabelas 1 e 2 mostram que, para o grupo monofocal, 15\% dos olhos (6) são hipermétropes, 75\% (30) são míopes e 10\% (4) são planos. Para o grupo das lentes multifocais, 20\% (8) são hipermétropes, 50\% (20) são míopes e 30\% (12) são planos.

Para a comparação dos grupos, foram realizados testes de sensibilidade e ofuscamento (Tabelas 5 e 6), além da aferição da acuidade visual com e sem correção (Tabelas 3 e 4) para longe (olhos direito e olho esquerdo) e para perto (ambos os olhos).

As comparações da aferição da acuidade visual com e sem correção da visão para longe e perto mostraram que a diferença das médias não foram estatisticamente significantes nos grupos com correção. Entretanto, a diferença das médias foram estatisticamente significantes $(p<0,05)$ nos grupos sem correção e, portanto, os pacientes que utilizaram a lente multifocal (diferença entre as lentes de 0,12 para longe e 0,27 para perto) obtiveram melhores resultados na visão para longe e perto.

Os resultados referentes à acuidade visual sem correção da visão para perto nos dois grupos analisados foram: 75\% tiveram J1 no grupo multifocal contra 10\% do grupo monofocal; $95 \%$ dos pacientes obtiveram $\mathrm{J} 2$ ou melhor no grupo multifocal, enquanto que $40 \%$ do pacientes do grupo monofocal obtiveram J2 ou melhor; $100 \%$ dos pacientes das lentes multifocais obtiveram $\mathrm{J} 3$ ou melhor, contra $70 \%$ do grupo monofocal (Tabela 7).

No grupo monofocal a média do teste de sensibilidade ao contraste foi de $0,57(\mathrm{DP}=0,21)$ e de $0,51(\mathrm{DP}=0,18)$ no grupo multifocal, com $p$-value de 0,17 , não havendo evidências estatísticas de que a diferença das médias dos resultados aferidos pelo teste de sensibilidade ao contraste seja significativa $(\mathrm{p}>0,05)$.

A diferença da média dos resultados do teste de ofuscamento para os dois grupos é estatisticamente significativa $(\mathrm{p}<0,05$, com uma diferença de 0,08$)$ e, portanto, a acuidade visual dos pacientes que utilizaram a lente multifocal no teste de ofuscamento é menor do que a dos pacientes que usaram a lente monofocal.

\section{DISCUSSÃO}

O conceito da cirurgia da catarata está sendo cada vez mais discutido com objetivo refrativo, no intuito da emetropia total, 


\begin{tabular}{|c|c|c|c|c|}
\hline \multirow{2}{*}{$\begin{array}{l}\text { Grupo monofocal } \\
\text { Nome }\end{array}$} & \multicolumn{2}{|c|}{ Olho direito } & \multicolumn{2}{|c|}{ Olho esquerdo } \\
\hline & Refração para longe & Equivalente esférico & Refração para longe & Equivalente esférico \\
\hline 1 & Plano & 0,00 & $+0,25:-0,50 \times 180$ & 0,00 \\
\hline 2 & $-0,50:-0,50 \times 175$ & $-0,75$ & $0,50:-0,50 \times 160$ & $-0,75$ \\
\hline 3 & pl : $-1,25 \times 105$ & $-0,62$ & $\mathrm{pl}:-1,00 \times 75$ & $-0,50$ \\
\hline 4 & $-0,25:-1,00 \times 60$ & $-0,75$ & $-0,50:-1,00 \times 80$ & $-1,00$ \\
\hline 5 & $-0,50$ & $-0,50$ & $-0,50$ & $-0,50$ \\
\hline 6 & $+0,50$ & $+0,50$ & $-0,75$ & $-0,75$ \\
\hline 7 & $+0,25$ & $+0,25$ & $+0,25:-0,25 \times 35$ & $+0,12$ \\
\hline 8 & $+0,75:-0,25 \times 110$ & $-0,62$ & $\mathrm{pl}:-0,75 \times 80$ & $-0,37$ \\
\hline 9 & pl : $-0,25 \times 5$ & $-0,12$ & $\mathrm{pl}:-0,50 \times 10$ & $-0,25$ \\
\hline 10 & $-0,50:-0,75 \times 110$ & $-0,87$ & pl: $-1,00 \times 85$ & $-0,50$ \\
\hline 11 & $-0,25:-0,50 \times 65$ & $-0,50$ & $-0,75:-0,25 \times 15$ & $-0,87$ \\
\hline 12 & $+0,25:-0,50 \times 120$ & 0,00 & $+0,25$ & $+0,25$ \\
\hline 13 & $+0,50:-0,75 \times 60$ & $+0,12$ & $+0,25:-0,25 \times 110$ & $+0,12$ \\
\hline 14 & pl : $-0,25 \times 90$ & $-0,12$ & 0,50 & $-0,50$ \\
\hline 15 & pl : $-0,25 \times 150$ & $-0,12$ & $\mathrm{pl}:-0,50 \times 15$ & $-0,25$ \\
\hline 16 & $-0,75:-0,50 \times 55$ & $-1,00$ & $-0,50:-0,50 \times 140$ & $-0,75$ \\
\hline 17 & $0,50:-1,00 \times 85$ & $-1,00$ & pl : $-1,00 \times 90$ & $-0,50$ \\
\hline 18 & $\mathrm{pl}:-0,50 \times 100$ & $-0,25$ & $\mathrm{pl}$ & 0,00 \\
\hline 19 & $\mathrm{pl}:-0,50 \times 15$ & $-0,25$ & $-0,50:-0,75 \times 55$ & $-0,87$ \\
\hline 20 & $-0,50:-0,50 \times 75$ & $-0,75$ & $-0,75$ & $-0,75$ \\
\hline \multicolumn{5}{|c|}{ Média do equivalente esférico $=-0,58 \mathrm{D}$} \\
\hline
\end{tabular}

\begin{tabular}{|c|c|c|c|c|}
\hline \multirow{2}{*}{$\begin{array}{c}\text { Grupo multifocal } \\
\text { Nome }\end{array}$} & \multicolumn{2}{|c|}{ Olho direito } & \multicolumn{2}{|c|}{ Olho esquerdo } \\
\hline & Refração para longe & Equivalente esférico & Refração para longe & Equivalente esférico \\
\hline 1 & $-0,50:-1,00 \times 35$ & $-1,00$ & $-0,25:-0,50 \times 90$ & $-0,50$ \\
\hline 2 & $\mathrm{pl}$ & 0,00 & $-0,50:-0,50 \times 180$ & $-0,75$ \\
\hline 3 & $+0,25:-0,75 \times 105$ & $-0,12$ & $+1,00:-0,50 \times 65$ & $+0,75$ \\
\hline 4 & $\mathrm{pl}:-0,50 \times 140$ & $-0,25$ & $+0,25$ & $+0,25$ \\
\hline 5 & $\mathrm{pl}$ & 0,00 & pl : $-0,50 \times 170$ & $-0,25$ \\
\hline 6 & $\mathrm{pl}:-1,00 \times 165$ & $-0,50$ & $-0,50$ & $-0,50$ \\
\hline 7 & $\mathrm{pl}:-0,50 \times 140$ & $-0,25$ & $\mathrm{pl}:-0,50 \times 15$ & $-0,25$ \\
\hline 8 & $\mathrm{pl}$ & 0,00 & pl : $-0,50 \times 130$ & $-0,25$ \\
\hline 9 & $\mathrm{pl}$ & 0,00 & $-0,75$ & $-0,75$ \\
\hline 10 & pl : $-0,50 \times 27$ & $-0,25$ & $\mathrm{pl}:-1,00 \times 165$ & $-0,50$ \\
\hline 11 & $+1,00$ & $+1,00$ & $+0,50:-0,50 \times 95$ & $+0,25$ \\
\hline 12 & $+0,25:-0,50 \times 165$ & 0,00 & pl : $-0,50 \times 180$ & $-0,25$ \\
\hline 13 & $\mathrm{pl}$ & 0,00 & $\mathrm{pl}$ & 0,00 \\
\hline 14 & $\mathrm{pl}:-0,50 \times 35$ & $-0,25$ & $\mathrm{pl}:-0,75 \times 70$ & $-0,37$ \\
\hline 15 & $+0,25:-0,50 \times 120$ & 0,00 & $\mathrm{pl}:-0,50 \times 80$ & $-0,25$ \\
\hline 16 & $\mathrm{pl}$ & 0,00 & $\mathrm{pl}$ & 0,00 \\
\hline 17 & $+1,00$ & $+1,00$ & $\mathrm{pl}$ & 0,00 \\
\hline 18 & pl & 0,00 & $+0,75:-0,75 \times 5$ & $+0,37$ \\
\hline 19 & $-0,50:-0,50 \times 90$ & $-0,75$ & $-0,50$ & $-0,50$ \\
\hline 20 & $+0,25$ & $+0,25$ & $+0,75$ & $+0,75$ \\
\hline \multicolumn{5}{|c|}{ Média do equivalente esférico $=-0,10 \mathrm{D}$} \\
\hline
\end{tabular}

tentando proporcionar aos pacientes uma visão para perto e longe sem os óculos ${ }^{(4)}$.

Para proporcionar uma visão para longe, muitos cirurgiões utilizam lentes intra-oculares monofocais, selecionando uma lente intra-ocular para visão para longe num dos olhos e no olho contralateral uma lente intra-ocular com visão para perto - uma estratégia chamada de monovisão ou báscula. Entretanto, a monovisão perde a capacidade da visão binocular dentre elas, a estereopsia. A outra alternativa no que se refere à visão para perto é a lente multifocal ${ }^{(5)}$. 


\begin{tabular}{|c|c|c|c|c|c|c|c|c|}
\hline \multirow[b]{2}{*}{ Paciente } & \multicolumn{4}{|c|}{ Monofocal $\mathrm{N}=40$} & \multicolumn{4}{|c|}{ Multifocal $\mathrm{N}=40$} \\
\hline & $\begin{array}{c}\text { Avs/c } \\
\text { OD }\end{array}$ & $\begin{array}{c}\text { Avs/c } \\
\text { OE }\end{array}$ & $\begin{array}{c}\text { Avc/c } \\
\text { OD }\end{array}$ & $\begin{array}{c}\text { Avc/c } \\
\text { OE }\end{array}$ & $\begin{array}{c}\text { Avs/c } \\
\text { OD }\end{array}$ & $\begin{array}{c}\text { Avs/c } \\
\text { OE }\end{array}$ & $\begin{array}{c}\text { Avc/c } \\
\text { OD }\end{array}$ & $\begin{array}{c}\text { Avc/c } \\
\text { OE }\end{array}$ \\
\hline 1 & 1,00 & 1,00 & 1,00 & 1,00 & 0,80 & 0,80 & 1,00 & 1,00 \\
\hline 2 & 0,80 & 0,80 & 1,00 & 1,00 & 1,00 & 1,00 & 1,00 & 1,00 \\
\hline 3 & 1,00 & 1,00 & 1,00 & 1,00 & 1,00 & 1,00 & 1,00 & 1,00 \\
\hline 4 & 0,63 & 0,63 & 1,00 & 1,00 & 1,00 & 1,00 & 1,00 & 1,00 \\
\hline 5 & 0,63 & 1,00 & 1,00 & 1,00 & 1,00 & 1,00 & 1,00 & 1,00 \\
\hline 6 & 0,80 & 0,63 & 1,00 & 1,00 & 0,80 & 1,00 & 1,00 & 1,00 \\
\hline 7 & 1,00 & 1,00 & 1,00 & 1,00 & 1,00 & 1,00 & 1,00 & 1,00 \\
\hline 8 & 0,80 & 1,00 & 1,00 & 1,00 & 1,00 & 1,00 & 1,00 & 1,00 \\
\hline 9 & 1,00 & 1,00 & 1,00 & 1,00 & 1,00 & 1,00 & 1,00 & 1,00 \\
\hline 10 & 0,80 & 0,80 & 1,00 & 1,00 & 1,00 & 1,00 & 1,00 & 1,00 \\
\hline 11 & 0,80 & 0,63 & 1,00 & 1,00 & 0,80 & 0,80 & 1,00 & 1,00 \\
\hline 12 & 0,63 & 0,63 & 1,00 & 1,00 & 0,80 & 1,00 & 1,00 & 1,00 \\
\hline 13 & 0,63 & 0,63 & 1,00 & 1,00 & 1,00 & 1,00 & 1,00 & 1,00 \\
\hline 14 & 0,80 & 0,80 & 1,00 & 1,00 & 1,00 & 0,80 & 1,00 & 1,00 \\
\hline 15 & 1,00 & 1,00 & 1,00 & 1,00 & 1,00 & 1,00 & 1,00 & 1,00 \\
\hline 16 & 0,63 & 0,80 & 1,00 & 1,00 & 1,00 & 1,00 & 1,00 & 1,00 \\
\hline 17 & 0,63 & 0,80 & 1,00 & 1,00 & 0,63 & 1,00 & 1,00 & 1,00 \\
\hline 18 & 1,00 & 1,00 & 1,00 & 1,00 & 1,00 & 1,00 & 1,00 & 1,00 \\
\hline 19 & 1,00 & 0,63 & 1,00 & 1,00 & 0,63 & 0,63 & 1,00 & 1,00 \\
\hline 20 & 0,63 & 1,00 & 1,00 & 1,00 & 1,00 & 1,00 & 1,00 & 1,00 \\
\hline
\end{tabular}

\begin{tabular}{|c|c|c|c|c|}
\hline \multirow[t]{2}{*}{ Paciente } & \multicolumn{2}{|c|}{ Monofocal } & \multicolumn{2}{|c|}{ Multifocal } \\
\hline & Avs/c & Avc/c & Avs/c & Avc/c \\
\hline 1 & 0,63 & 1,00 & 1,00 & 1,00 \\
\hline 2 & 0,80 & 1,00 & 1,00 & 1,00 \\
\hline 3 & 0,80 & 1,00 & 1,00 & 1,00 \\
\hline 4 & 0,80 & 1,00 & 1,00 & 1,00 \\
\hline 5 & 0,40 & 1,00 & 1,00 & 1,00 \\
\hline 6 & 1,00 & 1,00 & 1,00 & 1,00 \\
\hline 7 & 0,40 & 1,00 & 1,00 & 1,00 \\
\hline 8 & 0,63 & 1,00 & 1,00 & 1,00 \\
\hline 9 & 0,50 & 1,00 & 1,00 & 1,00 \\
\hline 10 & 0,80 & 1,00 & 0,80 & 1,00 \\
\hline 11 & 0,50 & 1,00 & 0,80 & 1,00 \\
\hline 12 & 0,63 & 1,00 & 1,00 & 1,00 \\
\hline 13 & 0,63 & 1,00 & 1,00 & 1,00 \\
\hline 14 & 0,50 & 1,00 & 1,00 & 1,00 \\
\hline 15 & 0,80 & 1,00 & 1,00 & 1,00 \\
\hline 16 & 0,63 & 1,00 & 0,63 & 1,00 \\
\hline 17 & 0,50 & 1,00 & 0,80 & 1,00 \\
\hline 18 & 0,63 & 1,00 & 0,80 & 1,00 \\
\hline 19 & 1,00 & 1,00 & 1,00 & 1,00 \\
\hline 20 & 0,80 & 1,00 & 1,00 & 1,00 \\
\hline \multicolumn{5}{|c|}{$\begin{array}{l}\text { Avs/c= Acuidade visual sem correção; Avc/c= Acuidade visual com correção; } \\
\text { Com correção-monofocal: média } 1,00 ; \text { DP } 0,00^{x} \text { multifocal } 1,00 ; D P 0,00 \text {. p-value } \\
1,000 ; \text { Sem correção-monofocal: média } 0,67 ; \text { DP } 0,18^{x} \text { multifocal } 0,94 ; \text { DP } 0,11 . \\
\text { p-value } 0,001 \text {. }\end{array}$} \\
\hline
\end{tabular}

Os primeiros estudos comparativos entre a lente multifocal e monofocal foram direcionados à visão monofocal. Implanta-

\begin{tabular}{|c|c|c|c|c|}
\hline \multirow{3}{*}{ Paciente } & \multicolumn{4}{|c|}{ Teste de sensibilidade } \\
\hline & \multicolumn{2}{|c|}{ Monofocal } & \multicolumn{2}{|c|}{ Multifocal } \\
\hline & OD & OE & OD & OE \\
\hline 1 & 0,50 & 0,50 & 0,50 & 0,50 \\
\hline 2 & 1,00 & 1,00 & 0,40 & 0,50 \\
\hline 3 & 0,50 & 1,00 & 0,50 & 0,50 \\
\hline 4 & 0,50 & 0,50 & 0,50 & 0,50 \\
\hline 5 & 0,50 & 1,00 & 1,00 & 0,50 \\
\hline 6 & 0,50 & 0,50 & 0,50 & 0,40 \\
\hline 7 & 0,50 & 0,50 & 0,50 & 0,40 \\
\hline 8 & 0,50 & 0,50 & 0,40 & 0,40 \\
\hline 9 & 0,50 & 0,50 & 0,50 & 0,40 \\
\hline 10 & 1,00 & 1,00 & 0,40 & 1,00 \\
\hline 11 & 0,40 & 0,32 & 1,00 & 0,50 \\
\hline 12 & 0,50 & 0,40 & 0,50 & 0,50 \\
\hline 13 & 0,50 & 0,50 & 0,50 & 0,50 \\
\hline 14 & 0,40 & 0,40 & 0,50 & 0,50 \\
\hline 15 & 1,00 & 0,50 & 0,40 & 0,40 \\
\hline 16 & 0,40 & 0,50 & 0,50 & 1,00 \\
\hline 17 & 0,40 & 0,40 & 0,32 & 0,40 \\
\hline 18 & 0,50 & 0,50 & 0,40 & 0,32 \\
\hline 19 & 0,50 & 0,50 & 0,40 & 0,40 \\
\hline 20 & 0,50 & 0,50 & 0,40 & 0,50 \\
\hline
\end{tabular}

va-se uma lente multifocal em um dos olhos e no olho contralateral uma lente monofocal com o propósito de reduzir as diferenças interpessoais. Entretanto, os pacientes usam nor- 


\begin{tabular}{|c|c|c|c|c|}
\hline \multirow{3}{*}{ Paciente } & \multicolumn{4}{|c|}{ Teste de ofuscamento } \\
\hline & \multicolumn{2}{|c|}{ Monofocal } & \multicolumn{2}{|c|}{ Multifocal } \\
\hline & OD & OE & OD & OE \\
\hline 1 & 0,50 & 0,50 & 0,50 & 0,32 \\
\hline 2 & 0,50 & 1,00 & 0,40 & 0,32 \\
\hline 3 & 0,32 & 0,50 & 0,50 & 0,50 \\
\hline 4 & 0,40 & 0,50 & 0,50 & 0,50 \\
\hline 5 & 0,20 & 0,50 & 0,40 & 0,32 \\
\hline 6 & 0,50 & 0,50 & 0,40 & 0,32 \\
\hline 7 & 0,50 & 0,50 & 0,32 & 0,32 \\
\hline 8 & 0,50 & 0,50 & 0,32 & 0,32 \\
\hline 9 & 0,40 & 0,40 & 0,32 & 0,32 \\
\hline 10 & 0,50 & 0,50 & 0,20 & 0,32 \\
\hline 11 & 0,32 & 0,32 & 1,00 & 0,50 \\
\hline 12 & 0,40 & 0,40 & 0,20 & 0,40 \\
\hline 13 & 0,50 & 0,50 & 0,50 & 0,32 \\
\hline 14 & 0,40 & 0,40 & 0,40 & 0,32 \\
\hline 15 & 0,50 & 1,00 & 0,40 & 0,40 \\
\hline 16 & 0,40 & 0,40 & 0,50 & 0,50 \\
\hline 17 & 0,40 & 0,32 & 0,20 & 0,40 \\
\hline 18 & 0,40 & 0,40 & 0,32 & 0,20 \\
\hline 19 & 0,50 & 0,50 & 0,32 & 0,40 \\
\hline 20 & 0,50 & 0,50 & 0,40 & 0,40 \\
\hline
\end{tabular}

\begin{tabular}{|c|c|c|}
\hline \multirow{2}{*}{$\begin{array}{c}\text { AVSC } \\
\text { (Perto) }\end{array}$} & \multicolumn{2}{|c|}{ Grupos } \\
\hline & Monofocal & Multifocal \\
\hline J1 & $10 \%$ & $75 \%$ \\
\hline J2 & $30 \%$ & $20 \%$ \\
\hline J3 & $30 \%$ & $5 \%$ \\
\hline J4 & $20 \%$ & \\
\hline J5 & $10 \%$ & \\
\hline Total & $100 \%$ & $100 \%$ \\
\hline
\end{tabular}

malmente a visão binocular. O implante bilateral de lente multifocal melhora a profundidade de foco e a visão de contraste, comparada à visão monofocal ${ }^{(6)}$.

$\mathrm{Na}$ presente pesquisa, a acuidade visual para longe sem correção foi ligeiramente melhor no grupo multifocal $(0,94) \mathrm{em}$ relação ao grupo monofocal $(0,82)$, p-value 0,001 . Cem por cento dos pacientes tiveram acuidade visual sem correção para longe melhor de 0,63 , conforme mencionado nos critérios de inclusão. No grupo multifocal, 16 pacientes $(80 \%)$ e no grupo monofocal 6 pacientes $(30 \%)$ tiveram acuidade visual nos dois olhos de 1,0 (20/20) sem correção, medidos separadamente. A acuidade visual sem correção foi pior no grupo das lentes monofocais devido à maioria dos pacientes $(75 \%)$ terem ficado ligeiramente míopes (média de -0,58 D). Essa ocorrência se deu na escolha do grau da lente (tendência a miopização) ${ }^{(6-7)}$.

Já no grupo multifocal, $50 \%$ dos pacientes ficaram míopes, $30 \%$ ficaram emétropes e $20 \%$ com hipermetropia. Esses resultados devem-se ao fato do cálculo de o poder da lente ter sido realizado com o objetivo de deixar o paciente emétrope ou com uma leve hipermetropia. Estudos comprovam que usando esta estratégia (hipermetropia 0,1 a $0,5 \mathrm{D}$ ) melhora-se o potencial óptico da lente ${ }^{(2,4)}$.

$\mathrm{Na}$ acuidade visual para perto e longe com correção, todos os pacientes tiveram acuidade visual de $1,0(20 / 20)$ e 1,0 (J1), ou seja, não tiveram perdas de linha de visão, dados concordantes com a literatura ${ }^{(6)}$.

No presente estudo, $75 \%$ dos pacientes do grupo multifocal tiveram J1 e 100\% tiveram AV para perto, melhor ou igual a J3. Enquanto que no grupo monofocal, a AV para perto em $10 \%$ foi $\mathrm{J} 1$ e $70 \%$ até $\mathrm{J} 3$, dados concordantes com outros autores na literatura ${ }^{(5-6,8-9)}$.

Apesar da visão para perto de $\mathrm{J} 1$, muitos pacientes do grupo multifocal necessitavam de óculos, pois a leitura tornava-se cansativa, principalmente nos pacientes acima de 70 anos. Provavelmente essa ocorrência se deu pela perda do contraste $^{(6)}$. Por outro lado, em pesquisa sobre qualidade de vida nos pacientes com implantes multifocais bilaterais, $40 \%$ não necessitavam de óculos em nenhuma ocasião ${ }^{(7)}$.

Na presente investigação científica, avaliou-se a curva de sensibilidade ao contraste nas freqüências espaciais de 3,6 , 12,18 , não comparando freqüências espaciais isoladas. Isto corresponde a um gráfico geral da sensibilidade ao contraste sem comparações específicas de determinada freqüência espacial em cada grupo e não houve diferença estatisticamente significativa entre os grupos comparados, concordantes com trabalhos publicados ${ }^{(6,8)}$. Outros pesquisadores ${ }^{(6)}$ relataram que esta diminuição da visão, em baixas freqüências espaciais, parece não interferir de forma importante na função visual.

Algumas doenças como ceratoconjuntivite seca, leucoma, transplante de córnea, opacidades vítreas e outras doenças sistêmicas como diabetes podem ter redução de sensibilidade ao contraste e ofuscamento. Nesse caso, deve ser evitado o implante de lente multifocal ${ }^{(8)}$. Neste estudo, não foi incluído nenhum paciente que fosse portador dessas referidas doenças.

$\mathrm{O}$ teste de ofuscamento apresentou uma nítida piora nos dois grupos pesquisados, sendo mais acentuado no grupo das multifocais $(0,39)$ em relação ao grupo das monofocais $(0,47)$, dados estes estatisticamente significativos, confirmando resultados já publicados ${ }^{(10)}$.

Comparando-se o desempenho da AMO Array com lente monofocal de PMMA, a sensibilidade ao contraste sob ofuscamento é geralmente diminuída principalmente nas freqüências espaciais $0,5,1,3,22,8^{(11-12)}$.

Existe uma grande dificuldade na avaliação do Teste de Sensibilidade ao Contraste (TSC) e ofuscamento devido ao grande número de aparelhos e à falta de padronização entre os diversos tipos de testes encontrados na literatura. Um outro fator de alteração do TSC é a não correção do astigmatismo, que pode provocar uma diminuição considerável da acuidade visual de contraste ${ }^{(13)}$.

As lentes multifocais melhoram sensivelmente a visão para perto e aconselhável em pacientes jovens, principalmente com catarata unilateral, pois preservariam a visão para perto. Entretanto, estes pacientes jovens com catarata unilateral, freqüen- 
temente comparam a visão do olho operado com a visão do olho sem a catarata e ao perceberem a diferença muitas vezes ficam insatisfeitos. Da mesma maneira, jovens com catarata bilateral, que sempre enxergaram de perto, algumas vezes ficam insatisfeitos com a visão multifocal, apesar de estarem J1 para perto. Pacientes de personalidades perfeccionistas, que demandam acuidade visual perfeita, motoristas profissionais e aqueles que dirigem freqüentemente à noite relataram maior freqüência de problemas visuais indesejáveis com halos e "glare" noturno ${ }^{(2)}$.

Acredita-se que a lente multifocal é um avanço tecnológico importante para a substituição das funções do cristalino natural, ou seja, restabelecer a visão para longe, intermediária e para perto. Embora não tenha sido objeto de pesquisa, queixas referentes à visão subjetiva de halos e arcos noturnos podem estar relacionadas à lente multifocal. Sendo assim, estudos futuros deverão ser realizados a fim de elucidar melhor essas alterações subjetivas, após o implante da lente multifocal.

Em conclusão, comprovamos que a acuidade visual para longe com correção nos pacientes com implante multifocal foi semelhante a dos pacientes com implantes monofocais, embora a acuidade visual para perto no grupo em que foi implantado lente multifocal foi bastante superior ao grupo da lente monofocal. A sensibilidade ao contraste manteve-se semelhante nos dois grupos, já o ofuscamento ("glare test") no grupo multifocal foi maior que no grupo monofocal.

\section{ABSTRACT}

Purpose: To compare distant and near visual acuity between patients submitted to bilateral nonsimultaneous facoemulsification and implantation of multifocal or monofocal intraocular lenses (IOL). Methods: Twenty patients with bilateral multifocal IOL and 20 patients with monofocal IOL with best corrected visual acuity (BCVA) of 20/30 or better, in separate measurements, within the first 3 months after surgery. Distant and near visual acuities with and without correction, contrast sensitivity and glare test were registered. Results: The uncorrected distant visual acuity of the monofocal group had a mean of $0.82(\mathrm{SD} \pm 0.16)$ and $0.9(\mathrm{SD} \pm 0.12)$ in the multifocal group, $\mathrm{p}$ value 0,001 . Both groups had BCVA of 1 . In the multifocal group, 75\% had Jaegger (J) 1 and 100\% had J3 or better for uncorrected near visual acuity (UNVA). In the monofocal group $10 \%$ had $\mathrm{J} 1$ and $70 \%$ had $\mathrm{J} 3$ or better for UNVA. There was no significant difference between both groups regarding the contrast sensitivity test. Both groups had vision reduction in the glare test, which was more pronounced in the multifocal group. Conclusions: The corrected distant visual acuity in the multifocal and monofocal groups showed to be similar, although UNVA in the multifocal group was better than in the monofocal group. The contrast sensitivity test showed similar results in both groups, but the glare test showed more intense discomfort in the multifocal group.

Keywords: Visual acuity; Lens implantation/intraocular; Lenses/ intraocular; Phacoemulsification; Adult

\section{REFERÊNCIAS}

1. Kara-José N, Arieta CEL. Catarata. In: Rodrigues MLV. Oftalmologia clínica. Rio de Janeiro: Cultura Médica; 1992. p.288-302.

2. Steinert RF. How to use the new multifocal IOL: Review of ophthalmology Boston; Feb 1998. p.74-80.

3. Gamerman D, Migon HS. Inferência estatística: uma abordagem integrada.Rio de Janeiro: Instituto de Matemática da Universidade Federal do Rio de Janeiro; 1997. [Coleção Textos de Métodos Matemáticos].

4. Centurion V, Lacava AC, Caballero JC. A performance visual com lente intraocular multifocal refrativa AMO Array. Rev Bras Oftalmol 1999;58:353-9.

5. Gimbel HV, Sanders DR, Raanan MG. Visual and refractive results of multifocal intraocular lenses. Ophthalmology 1991;98:881-8.

6. Arens B, Freudenthaler N, Quentin CD. Binocular function after bilateral implantation of monofocal and refractive multifocal intraocular lenses. J Cataract Refract Surg 1999;25:399-404.

7. Javitt JC, Wang F, Trentacost DJ, Rowe M, Tarantino N. Outcomes of cataract extraction with multifocal intraocular lens implantation: functional status and quality of life. Ophthalmology 1997;4:589-99.

8. Vaquero-Ruano M, Encinas JL, Millan I, Hijos M, Cajigal C. AMO Array multifocal versus monofocal intraocular lenses: long-term follow-up. J Cataract Refract Surg 1998;24:118-23.

9. Brydon KW, Tokarewicz AC, Nichols BD. AMO Array multifocal lens versus monofocal correction in cataract surgery. J Cataract Refract Surg 2000;26:96-100.

10. Akutsu H, Legge GE, Luebker A, Lindstrom RL, Zabel RW, Kirby VM. Multifocal intraocular lenses and glare. Optom Vis Sci 1993;70:487-95.

11. Sasaki A. Initial experience with a refractive multifocal intraocular lens in a Japanese population. J Cataract Refract Surg 2000;26:1001-7.

12. Weghaupt H, Pieh S, Skorpik C. Visual properties of the foldable Array multifocal intraocular lens. J Cataract Refract Surg 1996;22 Suppl 2:1313-7.

13. Lacava AC, Ceturion V. Teste de sensibilidade ao contraste e teste de ofuscamento no paciente portador de catarata. Arq Bras Oftalmol 1999;62:38-43.

Nos artigos enviados para publicação, o nome dos autores e suas afiliações devem estar completos. Isso facilitará a indexação e os links com as bases de dados e o CV Lates. 